\title{
4-Phenyl Butyric Acid (PBA) Promotes Aggregate Formation in HEK 293 Cells Expressing Wild Type or Mutant Pulmonary Surfactant Protein C (SP- C)
}

C.-L. Na, ${ }^{*}$ G. A. Stewart, * and T. E. Weaver*

*Cincinnati Children's Hospital Medical Center, Division of Pulmonary Biology, 3333 Burnet Ave, Cincinnati, OH 45229

Pulmonary surfactant protein C (SP-C) is a hydrophobic lipid associated protein secreted by alveolar type 2 cells that reduces alveolar surface tension during gas exchange. Accumulation of mutant SP-C proproteins, SP-C $\mathrm{C}^{\Delta \text { exon4 }}$ or SP-C ${ }^{\mathrm{L} 188 \mathrm{Q}}$, in endoplasmic reticulum (ER) leads to ER stress that may contribute to the pathogenesis of interstitial lung disease. 4-phenyl butyric acid (PBA) is a chemical chaperone that can reduce retention of mutant and misfolded proteins in ER, thereby decreasing protein aggregation and accumulation-associated cell stress. In this study, we tested whether PBA reduces accumulation of wild type (WT) and mutant SP-C protein aggregates in HEK 293 cells stably expressed SP-C ${ }^{\text {WT197 }}$, SP-C ${ }^{\text {L188Q }}$, and SP-C ${ }^{\text {exon4 }}$ with or without PBA incubation.

Electron dense aggregates detected by electron microscopy localized to ER, perinuclear inclusions, and lysosomes of control HEK 293 cells expressing WT and mutant SP-C (Figure $1 \mathrm{~A}-1 \mathrm{C}$ ). At least $50 \%$ of aggregates detected in control HEK 293 cells were less than $0.2 \mu \mathrm{m}$ in diameter. In the presence of $1 \mathrm{mM}$ PBA, pronounced aggregate formation was detected for all three HEK 293 cell lines expressing WT and mutant SP-C (Figure 1D-1F). Aggregate distribution analyzed by categorized rank test determined that there was a significant increase in the number of aggregates greater than $0.2 \mu \mathrm{m}$ in PBA-treated HEK 293 cells when compared to control cells $(p<0.001)$. The increase in aggregate formation was primarily associated with an increase in the number of aggregates between 0.2 to $0.4 \mu \mathrm{m}$. Although aggregate formation was also increased for aggregates greater than $0.4 \mu \mathrm{m}$, they were not statistically significant.

In addition to pronounced aggregate formation, PBA-treated mutant SP-C expressing HEK 293 cells often had dilated ER cisternae and mitochondria compared to control cells. Localization of SP-C proprotein by immuno EM detected focal localization of SP-C proprotein to the ER lumen, perinuclear electron dense inclusions, multivesicular bodies, and lysosomes of PBA treated cells (Figure 2A-2C). These findings are consistent with Western blotting analyses in which PBA not only increased accumulation of mutant $\mathrm{SP}-\mathrm{C}^{\mathrm{L} 188 \mathrm{Q}}$ and $\mathrm{SP}-\mathrm{C}^{\Delta \text { exon4 }}$ proprotein in mutant SP-C expressing HEK 293 cells but also increased accumulation of wild type SP-C proprotein in HEK 293 cells expressing SP-C ${ }^{\text {WT197 }}$, suggesting that PBA facilitates stabilization of WT and mutant SP-C proproteins.

These results suggest that PBA does not relieve aggregations in HEK 293 cells expressing WT and mutant SP-C. In contrast, an increase in stabilization of WT and mutant SP-C proproteins induced by PBA leads to elevated levels of SP-C proproteins and cytotoxicity in transfected HEK 293 cells. How PBA interacts with ER and other compartments to stabilize WT and mutant SP-C proproteins will require further investigation. 

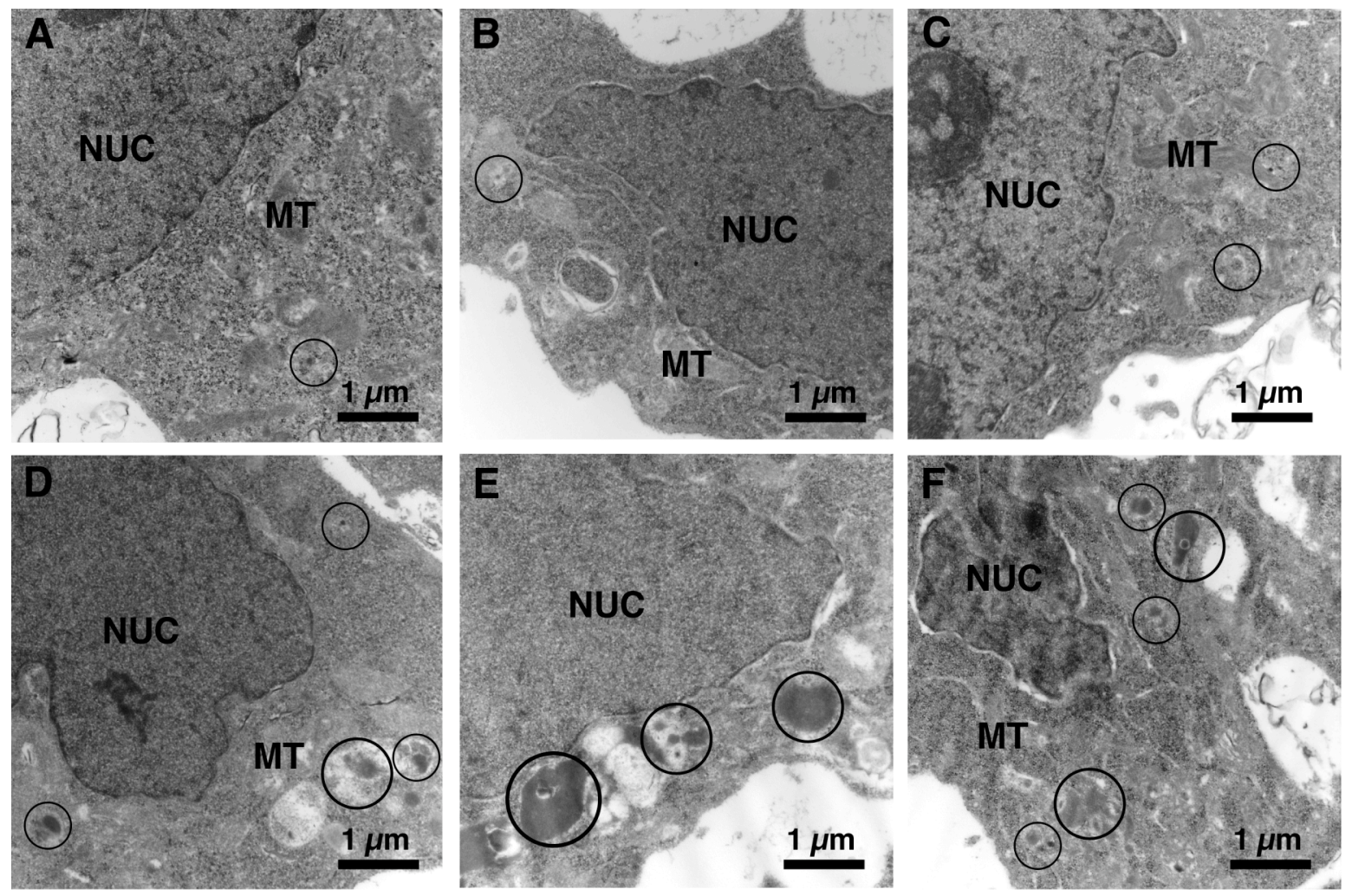

Figure 1. Localization of aggregates to HEK 293 cells expressing SP-C ${ }^{\mathrm{WT} 197}$, SP-C ${ }^{\mathrm{L} 188 \mathrm{Q}}$ and SP$\mathrm{C}^{\Delta \text { exon4 }}$ expressing HEK 293 cells with or without PBA. A. SP-C ${ }^{\text {WT197 }}$, buffer control. B. SP$\mathrm{C}^{\mathrm{L} 188 \mathrm{Q}}$, buffer control. C. SP-C ${ }^{\text {exon4}}$, buffer control. D. SP-C ${ }^{\mathrm{WT} 197}, 1 \mathrm{mM}$ PBA. E. SP-C ${ }^{\mathrm{L} 188 \mathrm{Q}}, 1$ $\mathrm{mM}$ PBA. F. SP-C $\mathrm{C}^{\Delta \text { exon4}}, 1 \mathrm{mM}$ PBA. Note that there was a significant increase in aggregate formation (circle) in PBA treated HEK 293 cells. NUC: nucleus; MT: mitochondria.
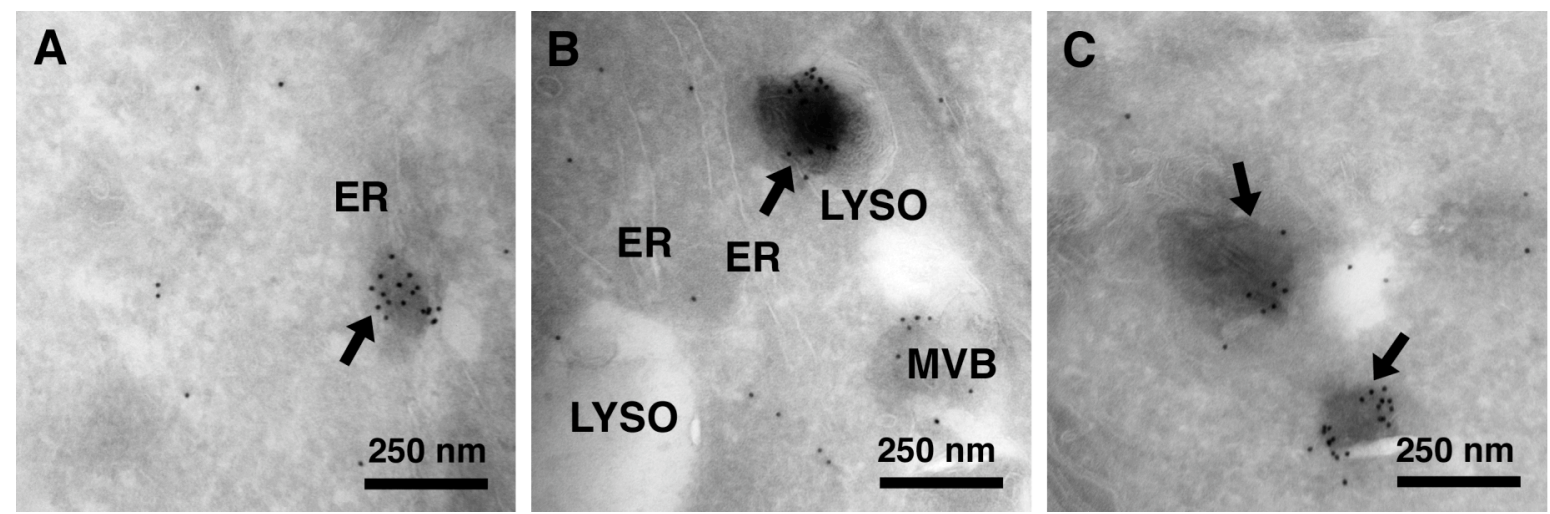

Figure 2. Localization of SP-C proprotein to $1 \mathrm{mM}$ PBA-treated HEK 293 cells expressing SP$\mathrm{C}^{\mathrm{WT1} 197}, \mathrm{SP}-\mathrm{C}^{\mathrm{L} 188 \mathrm{Q}}$, and SP-C ${ }^{\text {4exon4 }}$. A. HEK 293 cells expressing SP-C ${ }^{\mathrm{WT} 197}$. B. HEK 293 cells expressing SP-C ${ }^{\mathrm{L} 188 \mathrm{Q}}$. C. HEK 293 cells expressing SP-C ${ }^{\Delta \text { exon4}}$. Localization of SP-C proprotein was demonstrated by cryoimmunogold labeling using rabbit antisera directed against the Nterminus of SP-C proprotein and $10 \mathrm{~nm}$ protein A gold. Note that SP-C proprotein positive aggregates (arrow) localized to ER cisternae and perinuclear electron dense inclusions. ER: endoplasmic reticulm; LYSO: lysosome; MVB: multivesicular body. 